\title{
1 A test of the hierarchical model of litter decomposition
}

3 Mark A. Bradford, ${ }^{1,2^{*}}$, G. F. (Ciska) Veen, ${ }^{2}$ Anne Bonis, ${ }^{3}$ Ella M. Bradford, ${ }^{2}$ Aimee T.

4 Classen, ${ }^{4,5}$ J. Hans C. Cornelissen, ${ }^{6}$ Thomas. W. Crowther, ${ }^{7}$ Jonathan R. De Long, ${ }^{8}$ Gregoire T.

5 Freschet, ${ }^{9}$ Paul Kardol, ${ }^{10}$ Marta Manrubia-Freixa, ${ }^{2}$ Daniel S. Maynard, ${ }^{1}$ Gregory S. Newman, ${ }^{4,5}$

6 Richard S.P. van Logtestijn, ${ }^{6}$ Maria Viketoft, ${ }^{11}$ David A. Wardle, ${ }^{10,12}$ William R. Wieder, ${ }^{13}$

7 Stephen A. Wood, ${ }^{14}$ and Wim H. van der Putten ${ }^{2,15}$

$9 \quad{ }^{1}$ School of Forestry and Environmental Studies, Yale University, New Haven, CT 06511, USA

$10{ }^{2}$ Department of Terrestrial Ecology, Netherlands Institute of Ecology (NIOO-KNAW), $6700 \mathrm{AB}$

11 Wageningen, The Netherlands

12 3UMR 6553 ECOBIO - OSUR, University Rennes I - CNRS, Campus Beaulieu, Avenue du Gl

13 Leclerc, 35042 Rennes cedex, France

$14{ }^{4}$ The Rubenstein School, University of Vermont, 81 Carrigan Drive, Burlington, VT 05405, USA

$15{ }^{5}$ The Center for Macroecology, Evolution and Climate, Natural History Museum of Denmark,

16 University of Copenhagen, Universitetsparken 15, 2100 Copenhagen Ø, Denmark

17 Systems Ecology, Department of Ecological Science, Vrije Universiteit, De Boelelaan 1085,

181081 HV Amsterdam, The Netherlands

19 ' Institute of Integrative Biology, ETH Zurich, Univeritätstrasse 16, 8006, Zürich, Switzerland

$20{ }^{8}$ School of Earth and Environmental Sciences, The University of Manchester, Manchester M13

21 9PT, UK

$22{ }^{9}$ Centre d'Ecologie Fonctionnelle et Evolutive, UMR 5175 (CNRS - Université de Montpellier -

23 Université Paul-Valéry Montpellier - EPHE), 1919 route de Mende, Montpellier 34293, France

$24{ }^{10}$ Department of Forest Ecology and Management, Swedish University of Agricultural Sciences, 25 901-83 Umeå, Sweden

$26 \quad{ }^{11}$ Department of Ecology, Swedish University of Agricultural Sciences, P.O. Box 7044, 75007

27 Uppsala, Sweden

$28{ }^{12}$ Asian School of the Environment, Nanyang Technological University, 50 Nanyang Avenue,

29 Singapore 639798

$30 \quad{ }^{13}$ Climate and Global Dynamics Laboratory, National Center for Atmospheric Research,

31 Boulder, CO 80307, USA 
$32 \quad{ }^{15}$ The Nature Conservancy, Arlington VA, USA

$33{ }^{15}$ Laboratory of Nematology, Wageningen University, P.O. Box 8123, 6700 ES Wageningen,

34 The Netherlands

35

$36 \quad{ }^{*}$ Correspondence e-mail: mark.bradford@yale.edu 
37 Our basic understanding of plant litter decomposition informs the assumptions underlying

38 widely applied soil biogeochemical models, including those embedded in Earth system

39 models. Confidence in projected carbon cycle-climate feedbacks therefore depends on

40 accurate knowledge about the controls regulating the rate at which plant biomass is

41 decomposed into products such as $\mathrm{CO}_{2}$. Here, we test underlying assumptions of the

42 dominant conceptual model of litter decomposition. The model posits that a primary

43 control on the rate of decomposition at regional to global scales is climate (temperature and

44 moisture), with the controlling effects of decomposers negligible at such broad spatial

45 scales. Using a regional-scale litter decomposition experiment at six sites spanning from

46 northern Sweden to southern France - and capturing both within and among site variation

47 in putative controls - we find that contrary to predictions from the hierarchical model,

48 decomposer (microbial) biomass strongly regulates decomposition at regional scales.

49 Further, the size of the microbial biomass dictates the absolute change in decomposition

50 rates with changing climate variables. Our findings suggest the need for revision of the

51 hierarchical model, with decomposers acting as both local- and broad-scale controls on

52 litter decomposition rates, necessitating their explicit consideration in global

53 biogeochemical models.

55 The dominant conceptual model of litter decomposition posits that the primary controls on the

56 rate of decomposition are climate, litter quality and decomposer organisms ${ }^{1}$. These controls are

57 hypothesized to operate hierarchically in space, with climate and litter quality co-dominant at

58 regional to global scales ${ }^{2-4}$, and decomposers operating only as an additional local control whose

59 effect is negligible at broader scales ${ }^{5}$. Consequently decomposers have been omitted as controls 
60 from biogeochemical models, whereas a recent surge of interest in their inclusion has shown that

61 carbon-cycle projections depend strongly on whether and how microbial decomposers are

62 represented ${ }^{6-9}$. Yet evidence that microbial decomposers regulate decomposition rates at

63 regional- to global-scales, independent of climate variables such as temperature and moisture, is

64 generally lacking. One possibility for this lack of evidence is suggested by scaling theory, where

65 the influence of mechanisms that act locally can be obscured in emergent, broad-scale patterns ${ }^{10}$.

66 Pattern and scale has been described as the central issue in ecology, where the inherent

67 challenge to prediction and understanding lies in the elucidation of mechanisms, which

68 commonly operate at different scales to those on which the patterns are observed ${ }^{10}$. This scale

69 mismatch appears true for at least some ecosystem processes, such as plant productivity ${ }^{10,11}$.

70 Decomposition processes, also, are controlled by variables operating at finer scales than those at

71 which the variables are typically measured and evaluated ${ }^{1}$. For example, extensive empirical

72 support for the hierarchical model of litter decomposition has been provided through multi-site

73 climate gradient studies ${ }^{12-15}$. These multi-site studies have some common characteristics, which

74 include collecting few observations (typically 2 to 4 per site per litter species per collection) -

75 from which a mean decomposition rate is determined - and also use of site-mean data to estimate

76 climatic controls ${ }^{1}$. Yet the hierarchical model, and its representation in the structure of

77 biogeochemical models, is based on the assumption that controls act at the microsite level, by

78 regulating the activities of decomposer organisms ${ }^{5,16}$. That is, the hierarchical model is

79 conceptually grounded in local (i.e. microsite) dynamics, but has been developed and

80 substantiated with site-mean data that represents climate control of decomposition as an among-

81 site relationship. 
Understanding controls on litter decomposition across regional scales is then necessarily

83 intertwined with scaling theory. This body of theory ${ }^{10}$ suggests that broad-scale patterns might

84 emerge from distinct, local-scale causative relationships, which contrasts with the assumption of

85 the hierarchical model that among-site patterns in decomposition approximate patterns operating

86 at the microsite (Fig. 1). We refer to this as the "assumption of scale invariance" (Fig. 2a). Two

87 lines of evidence question the validity of the assumption of scale invariance for litter

88 decomposition. The first is that the activities of decomposer communities are shaped by

89 environmental selection for a subset of functional traits, which then uniquely dictate how

90 decomposition rates respond to changing climatic controls ${ }^{17-20}$. The second is that microclimate

91 can vary widely within a site ${ }^{21,22}$. As such, site-mean climate data are likely a poor surrogate for

92 the range in microclimate experienced by decomposer organisms within a site ${ }^{21}$. Both lines of

93 evidence support the possibility that among-site patterns in decomposition rates emerge from

94 distinct microsite-level relationships (the “assumption of scale dependence”, Fig. 2b).

95 We use a multi-site, litter decomposition study to test between the competing

96 assumptions of scale invariance and dependence (Figs. 1,2). We worked across a climate

97 gradient in Europe at six grassland sites spanning boreal climate in northern Sweden to

98 Mediterranean climate in southern France. We predicted two specific patterns would emerge if

99 the assumption of scale invariance were to be falsified. Prediction 1 was that relationships

100 between climate and decomposition rates should differ when site-mean versus microsite-level

101 climate data are analysed. That is, the emergent regional-scale pattern from microclimate data

102 should differ from the pattern observed with site-mean climate data. Prediction 2 was that any

103 variable expected to be an important control at the microsite-level (e.g. microbial biomass),

104 should have a strong effect when regional-scale patterns are analysed using microsite-level data. 
106 contrasting litter functional traits, but was not under test. Instead, standardizing known

107 controlling variables can improve estimated effects of other controls under study. In addition,

108 litter traits are expected to interact with controls such as temperature ${ }^{23}$ and so including this

109 variable allowed us to test this possibility. In total, we measured four controls (temperature,

110 moisture, microbial biomass and soil nitrogen availability) that naturally varied among

111 microsites. All four variables are expected to act as strong local and, in the case of the climate

112 variables, broad-scale controls on decomposition ${ }^{1,5,24,25}$. We then built a set of regression models,

113 structured to represent and test between assumptions of scale invariance versus dependence in

114 controls (see Methods), to compare the estimated effect sizes of these different variables on litter

115 decomposition rates.

117 Results and discussion

118 Decomposition rates varied within and among sites and between the two litter types (Fig. 3a,b).

119 As expected, mass carbon (C) loss over the 3-month field incubations was approximately twice 120 as great for the higher quality Holcus litter (33.8 $\pm 11.62 \%$; mean \pm SD) than for the Festuca litter

121 (16.8 $\pm 7.15 \%)$. However, there was considerable variation, with loss rates for Holcus ranging

122 from 7.72 to $53.7 \%$, and for Festuca from 0.50 to $35.3 \%$. Similarly there was marked variation in

123 the values of the climate controls, temperature and moisture, although they had contrasting

124 within versus among site distributions. Soil temperatures clustered within sites, meaning that

125 variation was much greater among sites (Fig. 3c), ranging from 10.0 to $25.3^{\circ} \mathrm{C}$ for the most

126 northern to southern site means. In contrast, microsite litter moisture only clustered around the

127 site mean at the two most southern sites, where mean site moisture was lowest (11.7 and 7.5\%). 
128 At the most northern site the mean moisture was 51.6\% but varied among microsites from 12.8

129 to $81.3 \%$ (Fig. 3d). Microsite soil nitrogen (N) availability and microbial biomass were more

130 clustered than moisture but within- versus among-site variation was still large (Figs. 3e,f). Soil N

131 varied among sites from means of 9.0 to $32.8 \mu \mathrm{g} \mathrm{N}$ g soil ${ }^{-1}$ but within the most northern site

132 alone from 2.3 to $70.6 \mu \mathrm{g} \mathrm{N}$ g soil ${ }^{-1}$. Equally, microbial biomass site means varied 2 -times from

1330.96 to $2.03 \mu \mathrm{g} \mathrm{CO}_{2} \mathrm{~g}$ soil $^{-1} \mathrm{~h}^{-1}$, but within sites from about 1.6-times (most northern) to about

134 2.75-times (most southern).

135 Prediction 1 was that emergent patterns between mean-site climate and decomposition

136 might fail to capture relationships occurring at the microsite scale. We found no support for this

137 prediction for temperature, with the “Microclimate” and “Site-mean climate” models (see

138 Methods) giving similar temperature coefficients (Table 1) and effect sizes (Fig. 4a). That is, the

139 temperature-decomposition relationship was scale invariant (Fig. 1). This perhaps is not

140 surprising given that microsite soil temperature clustered around the site mean (Fig. 3c).

141 Consequently the regional temperature-decomposition relationship should be, and was,

142 approximately equivalent whether microsite or site-mean values were explored (Fig. 4a). There

143 is evidence that microsite temperature can differ markedly to the site mean in some

144 environmental contexts ${ }^{22}$. However across 60 sites spanning a broad range in eco-climatic

145 conditions, Loescher et al. ${ }^{21}$ found that microsite soil temperatures were representative of the site

146 mean, suggesting that our finding that the temperature-decomposition relationship is scale

147 invariant might generalize to numerous ecosystem types.

148 In contrast, the moisture-decomposition relationship was strongly scale dependent: there 149 was a pronounced moisture-decomposition relationship for the Microsite model but a weak one 150 for the emergent pattern estimated from the Site-mean model (Table 1, Fig. 4b). Specifically, 
151 across the large observed range of microsite moisture availability (5.7 to 83.2\%), the Site-mean

152 model projected mass loss values ranging from a low of $27.4 \%$ to a high of $28.7 \%$. In contrast,

153 the Microclimate model estimated a shift in decomposition across the same range in moisture

154 from 23.9 to 33.2\% mass loss (Fig. 4b). Site means therefore poorly captured regional

155 heterogeneity in microsite moisture availability, generating a scale mismatch between local

156 mechanism and broad-scale pattern. Our data (Fig. 4b) consequently suggest that patterns

157 emerging from among-site comparisons of site-mean moisture may fail to represent causative

158 relationships operating at the much finer spatial scales at which decomposer organisms respond

159 to the environment. These findings raise questions about the use of site-mean (or coarser

160 resolution) hydroclimatic data to parameterise ecosystem models. Overall, our data suggest that

161 assumptions of the hierarchical model about scale invariance in climatic control are variable

162 dependent, cautioning against its general application as a conceptual and numerical

163 representation of controls on decomposition.

164 Using the "Microsite interactions" model (see Methods), we evaluated Prediction 2 that

165 variables considered locally important should retain a strong influence at broad spatial scales.

166 Following this prediction, the effect size of microbial biomass on decomposition rates was of

167 similar magnitude to those for the climatic variables (Fig. 5a). Specifically, estimated

168 decomposition rates varied by $\sim 16 \%$ mass $C$ loss with temperature change, $\sim 11 \%$ with moisture

169 change, and $\sim 12 \%$ with microbial biomass change (Fig. 5a). Not surprisingly, given that we

170 experimentally generated marked differences in litter quality, estimated mass loss increased

$171 \sim 24 \%$ (from 17 to 41\%) with increasing initial litter N (Fig. 5a). The soil N effect size was by

172 contrast small, leading to about a $2 \%$ positive change in estimated mass $\mathrm{C}$ loss but, as with all the

173 other variables, the main effect coefficient was significant $(P<0.05$; Table 1, Fig. 5a). Although 
174 some 2-way interaction coefficients were of comparable or greater magnitude to the main effects

175 for temperature, moisture and microbial biomass (Table 1), qualitatively the estimated effect

176 sizes of these variables from the Microsite interactions and Microsite main effects models were

177 similar (Figs. 4, 5b). That is, when interactions were removed, litter quality, temperature,

178 moisture and microbial biomass all retained strong control on decomposition at the regional scale

179 of our study (Table 1, Supplementary Fig. 1).

180 Exclusion of soil animal decomposers does alter litter decomposition rates in at least

181 some biomes ${ }^{15,24,26-28}$ but microbial effects were not explicitly examined. However, the

182 representation of microbial biomass or growth in biogeochemical models can improve predictive

183 power ${ }^{9,29}$ and such variables are argued to relate most directly to spatial and temporal variation in

184 biogeochemical process rates ${ }^{7,8,30}$. In support of these arguments, the absolute size of our

185 estimated effects of microclimate on decomposition depended strongly on microbial biomass.

186 Specifically, using the Microsite interactions model we set microbial biomass at five values

187 representing the observed range of microsite variation, and then varied temperature and moisture

188 (Fig. 5c,d). Higher microbial biomass values generated a much greater absolute change in

189 decomposition rates with increasing temperature or moisture (Fig. 5c,d). For example, estimated

190 mass loss rates across the microsite moisture range only varied by $\sim 5 \%$ in absolute terms when

191 microbial biomass was low, to as much as 25\% (from 28.5 to $54.2 \%$ mass loss) when it was

192 high. This influence of microbial biomass was primarily additive given that, when it was dropped

193 from the modelling (giving the Microclimate model), there was minimal influence on the relative

194 effect sizes of litter quality, temperature and moisture (Fig. 4, Table 1). An outstanding question

195 is whether the microbial traits selected by a site's climatic context ${ }^{17,18}$ in turn influence the

196 magnitude of microclimate effects on decomposition, as is similarly observed through climate 
197 selection of plant functional traits ${ }^{23,31}$. Nevertheless, our data do support emerging numerical

198 frameworks showing that explicit representation of microbes as controlling variables can

199 dramatically change expected effects of climate on broad-scale decomposition dynamics ${ }^{6,8,32}$.

200 We found positive but relatively weak effects of soil $\mathrm{N}$ availability on decomposition

201 (Supplementary Fig. 1), despite the fact stoichiometry is considered a key control on microbial

202 growth efficiencies and hence biogeochemical process rates ${ }^{33-36}$. The effects might have been

203 stronger had the litter been of lower quality (e.g. <1\% initial $\mathrm{N}$ ), requiring microbes to source $\mathrm{N}$

204 from the environment for growth and enzyme production ${ }^{35}$. Such possibilities emphasize the fact

205 that the effect sizes we report are specific to the spatial and temporal scale of our study. For

206 example, the relative effect size of controls changes with how progressed litter decay is ${ }^{37-39}$.

207 Future work will need to test whether the hierarchical model can approximate controls on later

208 decomposition stages, in other biomes and at even broader spatial scales ${ }^{37,38}$, when challenged

209 with microsite data. Where the model cannot approximate controls (i.e. where broad-scale

210 emergent patterns do not reflect microsite relationships), new microsite-level studies will be

211 needed to re-estimate parameter values for important controls. Such studies should test whether

212 measuring fine-scale temporal as well as spatial variation might also necessitate a re-evaluation

213 of how decomposition rates are controlled. Notably, our study leaves unresolved how microsite

214 variation in litter quality might influence the nature of this co-dominant control. Further, it

215 suggests a need to re-design multi-site litter decomposition studies but does not address the

216 challenge of making these studies practical given the very large number of observations

217 apparently required to test when and to what extent emergent broad-scale patterns fail to capture

218 microsite-level mechanisms ${ }^{1}$. 
We acknowledge that three aspects of our design may have influenced our findings:

220 enclosing litter in mesh can alter the microclimate ${ }^{40}$; the litter species do not occur at every site;

221 and the microsite scale we measured may also be mismatched with the litterbag scale of the

222 response variable ${ }^{41}$. However, these caveats also apply to the multi-site litter decomposition

223 experiments that have helped build and reinforce the hierarchical model ${ }^{12-15}$. The important

224 caveat that we remove from these previous studies is the assumption that aggregate (i.e. site-

225 mean) data accurately capture the relationships between decomposition and the variables

226 regulating it that operate at local (microsite) scales. Notably, there is growing evidence that C-

227 and N-cycling processes in soil are driven to a large extent by microsite variation in controlling

228 variables across landscape to regional scales ${ }^{42-44}$. Those working in population and community

229 ecology have wrestled with the insight that aggregate data may not represent local behaviour and

230 hence lead to false conclusions and projections ${ }^{45}$; it seems the same insight may need to be

231 grappled with in ecosystem ecology.

232

\section{Conclusions}

234 Scaling theory in ecology describes how emergent patterns can arise from distinct and causative

235 relationships operating at finer-scales ${ }^{10}$. However, the issue is nested within a broader inferential

236 challenge traditionally debated in the social sciences and increasingly so in the natural

237 sciences ${ }^{42,46-48}$. Although apparently named without reference to the field of ecology, the issue is

238 termed "ecological inference” and refers to the process of using aggregate data to draw

239 conclusions about individual-level behaviour ${ }^{48}$. Causative relationships inferred from aggregate

240 data often fail to represent the variables that control how individuals respond to and act on the

241 environment ${ }^{49}$. By comparison, relationships inferred from site-mean data in regional- to global- 
242 scale litter decomposition experiments may operate locally, or instead emerge from a set of

243 distinct local-scale relationships and controlling variables. We have referred to these two

244 possibilities as the assumption of scale invariance versus scale dependence (Fig. 2). Although we

245 find temperature control scale invariant, our findings for moisture and microbial biomass control

246 suggest that the hierarchical model may be the product of a logical inference fallacy. That is, it

247 arises because aggregate data are falsely assumed to represent finer-scale causative

248 relationships ${ }^{42,48,49}$. Encouragingly, the rich body of work on scaling theory and the ecological

249 inference fallacy ${ }^{50}$ provides a platform for ecosystem ecology to test and potentially reformulate

250 its conceptual and numerical models used to explain and predict how biogeochemical processes

251 respond to a changing environment. Our findings help reinforce calls to test and reconsider

252 which environmental variables predominantly regulate biogeochemical process rates at regional-

253 to global-scales, and when doing so emphasize the need to work at the microsite scales at which

254 organisms perceive the environment.

255

256 Methods

257 Experimental design. Site layout. Our research was conducted in grasslands spanning $\sim 20^{\circ}$

258 latitude in Western Europe (Fig. 1). At each of six study sites, we established four 30-m linear

259 transects between $50 \mathrm{~m}$ and up to $2 \mathrm{~km}$ apart. Transects were chosen to capture within-site

260 heterogeneity in microclimate and land-use intensity (e.g. with or without grazing). Along each

261 transect we established $20 \times 20 \mathrm{~cm}$ quadrats at 5-m intervals, resulting in 7 quadrats per transect.

262 In the context of this study, 'quadrat' serves as the 'microsite scale'. Between 28 April and 16

263 May 2015, we placed two nylon mesh bags $(5 \times 10 \mathrm{~cm}$; mesh size $0.9 \times 1 \mathrm{~mm})$ at each quadrat, 10

$264 \mathrm{~cm}$ apart. The mesh size presumably minimized the effect of larger soil fauna (e.g. earthworms) 
265 on decomposition rates, and so our decomposition rates were likely primarily the product of 266 microbes and micro- and mesofauna ${ }^{24,40}$. Each mesh bag contained $1 \mathrm{~g}$ air-dried grass foliar litter

267 of either Holcus lanatus L. or Festuca rubra L., which differ in their litter chemical properties

268 (see below). This resulted in a total of 6 locations $\times 4$ transects $\times 7$ quadrats $\times 2$ litter types $=336$

269 litterbags. Litterbags were placed flush with the soil surface, within the existing litter layer and

270 were retrieved after $\sim 3$ months. Of the 336 bags placed, 32 were lost in the field to such events

271 as consumption by cows and accidental site mowing. The litter used to fill the litterbags was

272 collected as freshly senesced material in grasslands local to the Dutch site.

273

274 Leaf litter. Mean litter properties for H. lanatus versus F. rubra were pH of 6.12 vs. 5.61, \%N of 2751.78 vs. 1.03, C:N of 24.7 vs. 43.7, and lignin, calcium, magnesium and potassium contents of 276157 vs. 175 , 3.72 vs. 2.75 , 1.31 vs. 0.79 , and 6.55 vs. $1.50 \mathrm{mg} \mathrm{g}^{-1}$, respectively. That is, 277 regardless of the chemical property measured, $H$. lanatus was always less recalcitrant. By 278 including the two contrasting litter types at every site, we generated equal within and among site 279 variation in this variable. Doing so provided a statistical control whereby the strong within-site 280 litter type effect should be approximated by the among site effect, and so generate a scale 281 invariant pattern (Fig. 2a). Second, standardizing known controlling variables can improve 282 estimated effects of the controls under study (e.g. microclimate). Third, litter traits are expected 283 to interact with other variables, such as temperature ${ }^{23}$, and so including this variable allowed us 284 to test this possibility.

286 Measurements. Field. At each quadrat we determined microclimate at the start, after $\sim 6$ weeks 287 and at the end of the field incubation period. We collected three measures per quadrat and time 
288 point of soil temperature at 5-cm depth using a hand-held thermometer. Such repeated spot 289 measurements are effective at characterizing relative variation in microclimate ${ }^{42}$, and so our 290 measures are not indicative of absolute values experienced by the decomposing litters but instead 291 capture generally warmer vs. cooler microsites, or drier vs. wetter, across the course of the study. 292 At the mid and end time point, soil moisture content was determined gravimetrically in three soil 293 cores (5 cm depth, $2 \mathrm{~cm}$ diam.) from each quadrat; cores were pooled and dried at $105^{\circ} \mathrm{C}$ until 294 constant mass. We had intended to use these measures (plus initial soil moisture) to estimate 295 microsite moisture conditions, but marked differences in soil texture from clay (Umeå) to loamy 296 sand (Wageningen) meant that soil gravimetric moisture was a poor surrogate for litter layer 297 moisture conditions. Instead, we used litter moisture values (see Testing Prediction 1 below). 298 Additionally, at the start point of the field incubations, 8-10 soil cores of the same size were 299 taken and pooled per quadrat and were used to determine soil gravimetric moisture, microbial 300 biomass and $\mathrm{N}$ availability. Initial soil samples and retrieved litterbags were shipped to the 301 Netherlands Institute of Ecology to ensure common processing. Collectively these measures 302 were intended to give estimates of four variables identified as important controls of 303 decomposition either at broad-scales (i.e. temperature and moisture), or at local-scales (i.e.

304 microbial biomass and $\mathrm{N}$ availability) $)^{32,35,51,52}$. For soil microbial biomass, it is probably fairer to 305 consider this an estimate of the spatial variation in soil community activity, which includes 306 invertebrate decomposers, many of which will have been able to access the litter ${ }^{24,40}$, and 307 potentially also microbes not involved in litter decomposition. 
310 fresh and after drying at $65^{\circ} \mathrm{C}$. It was next milled to a fine powder and analysed for total C

311 content through elemental analysis (Flash 2000, Thermo Fisher Scientific, Bremen, Germany).

312 The initial 168 soils (6 locations $\times 4$ transects $\times 7$ quadrats) were passed through a 4-mm

313 sieve and sub-sampled for gravimetric moisture, microbial biomass and $\mathrm{N}$ availability. We used

314 the substrate-induced respiration (SIR) method to estimate active microbial biomass ${ }^{53}$, modified

315 per Fierer et al. ${ }^{54}$. We estimated soil $\mathrm{N}$ availability by determining potential net $\mathrm{N}$ mineralization

316 rates as the difference between salt-extractable $\mathrm{N}^{-} \mathrm{NO}_{3}{ }^{-}$and $\mathrm{N}-\mathrm{NH}_{4}{ }^{+}$at time zero and after $14 \mathrm{~d}$ of

317 incubation at $20^{\circ} \mathrm{C}$ and $65 \%$ water holding capacity ${ }^{55}$. Soils were extracted with $1 \mathrm{M} \mathrm{KCl}$ and

318 extracts measured using an auto-analyser (QuAAtro Segmented Flow Analyser; SEAL

319 Analytical; Norderstedt, Germany).

320 Initial litter properties were estimated using seven randomly collected samples per

321 species, matching the sub-sampling for the litterbags. Total $\mathrm{C}$ and $\mathrm{N}$ content were measured as

322 described above, lignin after a chloroform/methanol extraction and hydrolysis with $\mathrm{HCl}$,

323 following Poorter \& Villar ${ }^{56}$. Mineral nutrient concentrations and $\mathrm{pH}$ were measured following

324 methods described in Hendry and Grime ${ }^{57}$ and Cornelissen et al. ${ }^{58}$, respectively.

325

326 Data and inferential analysis. Overview of approach. We built a set of regression models,

327 structured to represent and test between assumptions of scale invariance versus dependence in 328 controls on litter decomposition (Fig. 2), to compare estimated effect sizes on decomposition of 329 the four controlling variables under study. Specifically, we estimated the relative effect size for 330 temperature, moisture, soil $\mathrm{N}$ availability and microbial biomass, across the range of observed 331 values within and among our six sites. The relative effect size depends on the slope coefficient 
332 for the specific variable, the slope coefficient for any interaction it is involved in, and the range

333 of observed values of the variable. We generated the coefficients by fitting linear mixed-effect

334 models (LMMs). The effect size of a variable on mass C loss was estimated using these

335 regression parameters, while holding all other variables constant (i.e. the mean of all

336 observations for each variable), and systematically varying the variable of interest across its

337 measured range of values. That is, we plotted the regression equation for a model using the

338 coefficients from the respective LMM, the mean value across all 168 quadrats for the controls

339 not under test, and then for the control under test we estimated decomposition rates by

340 systematically increasing the value of the control from the lowest to highest observed values

341 across the 168 quadrats.

342 The choice of variables to measure and then include in our statistical models (described

343 next) was based on the approach of Hobbs et al..$^{59}$, which rejects model selection on

344 philosophical and operational grounds. Philosophically, we investigated only variables where

345 biological mechanism as to their influence on decomposition is firmly established. Operationally,

346 there is subjectivity and lack of agreement in statistical model selection approaches, with

347 different decisions leading to markedly different conclusions as to effect sizes. Instead,

348 coefficients and hence effect sizes are generally most robust when all terms are retained,

349 assuming that each is included with well-established biological foundation.

350

351 Testing Prediction 1. Prediction 1 was that relationships between climate and decomposition

352 rates should differ when site-mean versus microsite-level climate data are analysed. This

353 prediction was evaluated by comparing whether temperature and moisture effects on mass C loss

354 differed when the slope coefficients were estimated from microsite versus site-mean data. We 
355 established a single model structure to test Prediction 1. It included only recognized broad-scale

356 controls as variables (i.e. temperature, moisture and litter type), but involved different data

357 aggregation. The "Microclimate" model was tested with observations of mass C loss for each

358 litterbag and quadrat-level microclimate. The "Site-mean climate” model was also run with all

359 litterbag observations - to minimize changes in predictive power associated with changing

360 values of $n$ - but the values of the climate variables were the mean per site of the microclimate

361 (i.e. quadrat) observations. Hence in the Microclimate model the dataset had 168 unique

362 temperature and moisture observations, whereas in the Site-mean climate model there were only

363 six possible values (one per site) of temperature and moisture. Specifically, microsite control

364 values were determined from the quadrat-level measures, and site mean values determined from

365 the mean of the 28 quadrat-measures within a site (i.e. they were based on the exact same set of

366 measurements). To account for potential spatial auto-correlation among the quadrats within a

367 site, we fit a random error structure accounting for the spatial hierarchy in the design (quadrat

368 nested within transect, with transect nested within site), assuming a common slope but spatially-

369 dependent intercept ${ }^{50,60}$.

370 Similarly, litter type was included as the litterbag-level \%N value, or as the mean \%N per

371 litter type, respectively (note that climate effect sizes were independent of how litter type was

372 included). To determine a litterbag-level initial \%N value, we randomly assigned to each

373 litterbag a \%N value (to the nearest $0.1 \%$ ) drawn from the measured range of initial \%N values

374 from seven additional litterbag samples (Fig. 3b). We did this to acknowledge that there was

375 variation among litterbags in initial \%N and so using the mean initial \%N would give a false

376 account of the among-bag variation. For quadrat-level temperature, we calculated the mean soil

377 temperature across the three field measurement periods. For quadrat-level moisture, given that 
378 soil gravimetric moisture was not useful given soil texture differences among sites, we calculated

379 quadrat-level moisture as the mean of the Holcus and Festuca litterbag moisture values on

380 collection. We acknowledge that litters were probably drier at collection than at earlier points of

381 the field incubations, given increasing temperatures and declining precipitation across the

382 incubations, and so these values provide an estimate of relative spatial differences in moisture

383 only. We used the mean across the two litter types, given that species-specific moisture values

384 are often a product of leaf litter traits and are thus correlated with litter quality ${ }^{4}$.

385

386 Testing Prediction 2. Prediction 2 was that any variable expected to be an important control at

387 the microsite-level, should have a strong effect when regional-scale patterns are analysed using

388 microsite data. Specifically, we evaluated whether effect sizes of the soil microbial biomass and

$389 \mathrm{~N}$ availability variables had effect sizes comparable to recognized broad-scale controls

390 (specifically temperature and moisture). We developed three model structures. The "Microsite

391 interactions” model included all variables (i.e. temperature, moisture, microbial biomass, $\mathrm{N}$

392 availability) and their 2-way interactions. We included two-way interactions among the main

393 effects given expectations that the relative effects of our variables should depend on one another.

394 For example, the decomposition rate of more recalcitrant litters is expected to be more

395 temperature sensitive ${ }^{61,62}$. The "Microsite main effects" model removed the 2-way interactions to

396 determine whether the effect sizes of the variables were primarily additive. The "Microclimate"

397 model was used again but to evaluate whether dropping the soil microbial biomass and $\mathrm{N}$

398 availability terms altered inferences about temperature and moisture controls on mass C loss.

399 Litter type (as initial \%N) was again included in all models.

400 
401 Statistical model specifics. The LMMs were fit with a Gaussian error distribution in the "Ime4"

402 package for the "R" statistical program (version 3.1.3), using the "Imer" function.

403 Decomposition was calculated as the proportional mass C loss from the litterbags. Site, transect 404 and quadrat were fit as random variables to the LMMs, with the finer scale variables nested 405 within the broader scale variables, given the potential for autocorrelation caused by spatially 406 clustering the litterbags ${ }^{60}$. Before we tested the model structures described above, we tested the 407 data distributions. A single and highly influential observation (based on Cook’s D) was dropped 408 from the dataset; it had a mass C loss value of 69.9\%, far higher than any other observation (Fig. 409 3a), and markedly affected residual fits. The remaining data conformed to assumptions of 410 normality, and a second-order temperature term was included given the observed unimodal 411 relationship between temperature and mass loss. Also, initial extractable $\mathrm{N}$ was a better choice 412 (i.e. higher standardized coefficient) than potential $\mathrm{N}$ mineralization for soil $\mathrm{N}$ availability, and 413 litter moisture (mean per quadrat) performed better than gravimetric soil moisture. Litter initial $414 \% \mathrm{~N}$ was used to represent litter quality given that it is a strong predictor of early-stage 415 decomposition in grasses such as H. lanatus ${ }^{39,63}$.

416 The square-root variance inflation factors (vif) were $<2$ for the main effects, indicating 417 low collinearity. As would be expected, there was a strong correlation between temperature and 418 its second-order term, and where the effect of one variable strongly interacted with another. We 419 reduced these 'vif' values by standardizing the observed value of each variable by subtracting the 420 mean and dividing by two standard deviations ${ }^{64}$. The resulting standardized coefficients also 421 permit coefficients to be directly compared for variables measured on different unit scales.

422 Confirming the validity of our inferences in spite of introduced collinearity when second-order 423 terms and interactions were permitted, variables with large effect sizes calculated on the basis of 
424 the unstandardized coefficients also had large standardized coefficients. In addition, in the

425 'Microsite main effects' model all 2-way interactions were dropped, removing collinearity and

426 concerns about over-fitting, and the relative magnitude of the coefficients were largely

427 unchanged (Table 1).

$428 \quad$ All reported $P$-values are quasi-Bayesian but retain the same interpretation as frequentist

$429 P$-values ${ }^{65}$. We considered coefficients with $P<0.05$ to be significant and coefficients with

$430 \quad P<0.10$ marginally significant. We calculated the $r^{2}$ values for each model following Nakagawa

431 and Schielzeth ${ }^{66}$. Calculation of $r^{2}$ values is common practice when modelling decomposition

432 and a high value associated with a specific explanatory variable is often associated with that

433 variable having a strong effect size. This reasoning makes no sense within the context of our

434 study because litter type was experimentally controlled and accurately measured within and

435 among sites, whereas the other variables relied on observed variation and measurements that

436 represented - but likely did not fully characterize - the conditions that acted on decomposer

437 activity. The latter conditions make data more "noisy", lowering $r^{2}$ values, but in the absence of

438 systematic bias will not change the coefficient estimates and hence effect sizes ${ }^{1}$. We therefore

439 only report the $r^{2}$ value for each model, to verify they had the potential to explain a substantive

440 degree of the variance in decomposition rate.

442 Data availability

443 Experimental data in the support of these findings and the R code for the statistical models are

444 available via the Dryad Digital Repository (http://dx.doi.org/10.5061/dryad.c44h0).

\section{References}


4471 Bradford, M. A., Berg, B., Maynard, D. S., Wieder, W. R. \& Wood, S. A. Understanding

448 the dominant controls on litter decomposition. J. Ecol. 104, 229-238 (2016).

4492 Cornwell, W. K. et al. Plant species traits are the predominant control on litter

450 decomposition rates within biomes worldwide. Ecol. Lett. 11, 1065-1071 (2008).

4513 Freschet, G. T., Aerts, R. \& Cornelissen, J. H. C. A plant economics spectrum of litter

452 decomposability. Func. Ecol. 26, 56-65 (2012).

4534 Makkonen, M. et al. Highly consistent effects of plant litter identity and functional traits

454 on decomposition across a latitudinal gradient. Ecol. Lett. 15, 1033-1041 (2012).

4555 Swift, M. J., Heal, O. W. \& Anderson, J. M. Decomposition in terrestrial ecosystems.

456 Studies in Ecology volume 5. (Blackwell Scientific, 1979).

4576 Buchkowski, R. W., Bradford, M. A., Grandy, A. S., Schmitz, O. J. \& Wieder, W. R.

458 Applying population and community ecology theory to advance understanding of

459 belowground biogeochemistry. Ecol. Lett. 20, 231-245 (2017).

4607 Sulman, B. N., Phillips, R. P., Oishi, A. C., Shevliakova, E. \& Pacala, S. W. Microbe-

461 driven turnover offsets mineral-mediated storage of soil carbon under elevated $\mathrm{CO}_{2}$. Nat.

462 Clim. Change 4, 1099-1102 (2014).

4638 Tang, J. \& Riley, W. J. Weaker soil carbon-climate feedbacks resulting from microbial

464 and abiotic interactions. Nat. Clim. Change 5, 56-60 (2014).

4659 Wieder, W. R., Bonan, G. B. \& Allison, S. D. Global soil carbon projections are

466 improved by modelling microbial processes. Nat. Clim. Change 3, 909-912 (2013).

46710 Levin, S. A. The problem of pattern and scale in ecology. Ecology 73, 1943-1967 (1992).

46811 Lauenroth, W. K. \& Sala, O. E. Long-term forage production of North American

469 shortgrass steppe. Ecol. App. 2, 397-403 (1992). 
47012 Berg, B. et al. Litter mass-loss rates in pine forests for Europe and Eastern United States:

471 some relationships with climate and litter quality. Biogeochem. 20, 127-159 (1993).

47213 Harmon, M. E. et al. Long-term patterns of mass loss during the decomposition of leaf

473 and fine root litter: an intersite comparison. Glob. Change Biol. 15, 1320-1338 (2009).

47414 Moore, T. R. et al. Litter decomposition rates in Canadian forests. Glob. Change Biol. 5, $475 \quad$ 75-82 (1999).

47615 Wall, D. H. et al. Global decomposition experiment shows soil animal impacts on

477 decomposition are climate-dependent. Glob. Change Biol. 14, 2661-2677 (2008).

47816 Bonan, G. B., Hartman, M. D., Parton, W. J. \& Wieder, W. R. Evaluating litter

479 decomposition in earth system models with long-term litterbag experiments: an example

480 using the Community Land Model version 4 (CLM4). Glob. Change Biol. 19, 957-974

$481 \quad$ (2013).

48217 Averill, C., Waring, B. G. \& Hawkes, C. V. Historical precipitation predictably alters the 483 shape and magnitude of microbial functional response to soil moisture. Glob. Change $484 \quad$ Biol. 5, 1957-1964 (2016).

48518 Strickland, M. S., Keiser, A. D. \& Bradford, M. A. Climate history shapes contemporary 486 leaf litter decomposition. Biogeochem. 122, 165-174 (2015).

48719 Fierer, N. et al. Cross-biome metagenomic analyses of soil microbial communities and 488 their functional attributes. P. Natl. Acad. Sci. USA 109, 21390-21395 (2012).

48920 Evans, S. E. \& Wallenstein, M. D. Climate change alters ecological strategies of soil $490 \quad$ bacteria. Ecol. Lett. 17, 155-164 (2014). 
49121 Loescher, H., Ayres, E., Duffy, P., Luo, H. \& Brunke, M. Spatial variation in soil

492 properties among North American ecosystems and guidelines for sampling designs. PLoS

$493 \quad$ One 9, e83216 (2014).

49422 Scherrer, D. \& Körner, C. Infra-red thermometry of alpine landscapes challenges climatic

495 warming projections. Glob. Change Biol. 16, 2602-2613 (2010).

49623 Meentemeyer, V. Macroclimate and lignin control of litter decomposition rates. Ecology

$497 \quad 59,465-472(1978)$.

49824 García-Palacios, P., Maestre, F. T., Kattge, J. \& Wall, D. H. Climate and litter quality

499 differently modulate the effects of soil fauna on litter decomposition across biomes. Ecol.

$500 \quad$ Lett. 16, 1045-1053 (2013).

50125 Tenney, F. G. \& Waksman, S. A. Composition of natural organic materials and their

502 decomposition in the soil: IV. The nature and rapidity of decomposition of the various

503 organic complexes in different plant materials, under aerobic conditions. Soil Science 28,

$504 \quad 55-84(1929)$.

50526 Handa, I. T. et al. Consequences of biodiversity loss for litter decomposition across

506 biomes. Nature 509, 218-221 (2014).

50727 Powers, J. S. et al. Decomposition in tropical forests: a pan-tropical study of the effects

508 of litter type, litter placement and mesofaunal exclusion across a precipitation gradient. $J$.

$509 \quad$ Ecol. 97, 801-811 (2009).

51028 Crowther, T. W. et al. Biotic interactions mediate soil microbial feedbacks to climate

511 change. P. Natl. Acad. Sci. USA 112, 7033-7038 (2015). 
51229 Lawrence, C. R., Neff, J. C. \& Schimel, J. P. Does adding microbial mechanisms of 513 decomposition improve soil organic matter models? A comparison of four models using 514 data from a pulsed rewetting experiment. Soil Biol. Biochem. 41, 1923-1934 (2009).

$51530 \quad$ Hall, E. et al. Understanding how microbiomes influence the systems they inhabit: 516 Insight from ecosystem ecology. bioRxiv http://dx.doi.org/10.1101/065128 (2016).

51731 Aerts, R. Climate, leaf litter chemistry and leaf litter decomposition in terrestrial 518 ecosystems: a triangular relationship. Oikos 79, 439-449 (1997).

51932 Allison, S. D., Wallenstein, M. D. \& Bradford, M. A. Soil-carbon response to warming 520 dependent on microbial physiology. Nat. Geosci. 3, 336-340 (2010).

52133 Crowther, T. W. et al. Environmental stress response limits microbial necromass 522 contributions to soil organic carbon. Soil. Biol. Biochem. 85, 153-161 (2015).

$523 \quad 34 \quad$ Frey, S. D., Lee, J., Melillo, J. M. \& Six, J. The temperature response of soil microbial 524 efficiency and its feedback to climate. Nat. Clim. Change 3, 395-398 (2013).

52535 Schimel, J. P. \& Weintraub, M. N. The implications of exoenzyme activity on microbial 526 carbon and nitrogen limitation in soil: a theorectical model. Soil Biol. Biochem. 35, 549$527 \quad 563(2003)$.

$52836 \quad$ Buchkowski, R. W., Schmitz, O. J. \& Bradford, M. A. Microbial stoichiometry overrides 529 biomass as a regulator of soil carbon and nitrogen cycling. Ecology 96, 1139-1149 $530 \quad$ (2015).

53137 Adair, E. C. et al. Simple three-pool model accurately describes patterns of long-term 532 litter decomposition in diverse climates. Glob. Change Biol. 14, 2636-2660 (2008). 
$533 \quad 38$ Currie, W. S. et al. Cross-biome transplants of plant litter show decomposition models

534 extend to a broader climatic range but lose predictability at the decadal time scale. Glob.

$535 \quad$ Change Biol. 16, 1744-1761 (2010).

53639 Smith, V. C. \& Bradford, M. A. Litter quality impacts on grassland litter decomposition 537 are differently dependent on soil fauna across time. Appl. Soil Ecol. 24, 197-203 (2003).

53840 Bradford, M. A., Tordoff, G. M., Eggers, T., Jones, T. H. \& Newington, J. E. Microbiota, 539 fauna, and mesh size interactions in litter decomposition. Oikos 99, 317-323 (2002).

54041 Bokhorst, S. \& Wardle, D. A. Microclimate within litter bags of different mesh size:

541 Implications for the 'arthropod effect' on litter decomposition. Soil Biol. Biochem. 58, $542 \quad 147-152(2013)$.

54342 Bradford, M. A. et al. Climate fails to predict wood decomposition at regional scales. $544 \quad$ Nat. Clim. Change 4, 625-630 (2014).

54543 Keiser, A. D., Knoepp, J. D. \& Bradford, M. A. Disturbance decouples biogeochemical $546 \quad$ cycles across forests of the southeastern US. Ecosystems 19, 50-61 (2016).

54744 Waring, B., Adams, R., Branco, S. \& Powers, J. S. Scale-dependent variation in nitrogen $548 \quad$ cycling and soil fungal communities along gradients of forest composition and age in 549 regenerating tropical dry forests. New Phyt. 209, 845-854 (2016).

55045 Schmitz, O. J. Resolving ecosystem complexity. (Princeton Univ. Press, 2010).

55146 Oakes, M. J. Commentary: individual, ecological and multilevel fallacies. Inter. J. $552 \quad$ Epidem. 38, 361-368 (2009).

55347 Robinson, W. S. Ecological correlations and the behavior of individuals. Am. Socio. Rev. $554 \quad$ 15, 351-357 (1950).

55548 Schuessler, A. A. Ecological inference. P. Natl. Acad. Sci. USA 96, 10578-10581 (1999). 
55649 Gelman, A., Shor, B., Bafumi, J. \& Park, D. Rich state, poor state, red state, blue state:

$557 \quad$ what's the matter with Connecticut? Qu. J. Poli. Sci. 2, 345-367 (2007).

$55850 \quad$ Gelman, A. \& Hill, J. Data analysis using regression and multilevel/hierarchical models. $559 \quad$ (Cambridge Univ. Press, 2007).

56051 Rousk, J. Biomass or growth? How to measure soil food webs to understand structure and 561 function. Soil Biol. Biochem. 102, 45-47 (2016).

56252 Allison, S. D. et al. Microbial abundance and composition influence litter decomposition 563 response to environmental change. Ecology 94, 714-725 (2013).

56453 Anderson, J. P. E. \& Domsch, K. H. A physiological method for the quantitative 565 measurement of microbial biomass in soils. Soil Biol. Biochem. 10, 215-221 (1978).

56654 Fierer, N., Schimel, J. P. \& Holden, P. A. Influence of drying-rewetting frequency on soil 567 bacterial community structure. Microb. Ecol. 45, 63-71 (2003).

$56855 \quad$ Robertson, G. P. et al. in Standard soil methods for long-term ecological research (eds G 569 P Robertson, D C Coleman, C S Bledsoe, \& P Sollins) 258-271 (Oxford University Press, $570 \quad$ 1999).

57156 Poorter, H. \& Villar, R. in Plant resource allocation (eds F A Bazzaz \& J Grace) 39-72 $572 \quad$ (Academic Press, 1997).

57357 Hendry, G. A. F. \& Grime, J. P. Methods in comparative plant ecology (Chapman \& $574 \quad$ Hall, 1993).

57558 Cornelissen, J. H. C. et al. Foliar $\mathrm{pH}$ as a new plant trait: can it explain variation in foliar $576 \quad$ chemistry and carbon cycling processes among subarctic plant species and types? $577 \quad$ Oecologia 147, 315-326 (2006). 
57859 Hobbs, N. T., Andren, H., Persson, J., Aronsson, M. \& Chapron, G. Native predators

579 reduce harvest of reindeer by Sámi pastoralists. Ecol. App. 22, 1640-1654 (2012).

$58060 \quad$ Bolker, B. M. et al. Generalized linear mixed models: a practical guide for ecology and $581 \quad$ evolution. Trends Ecol. Evol. 24, 127-135 (2009).

58261 Fierer, N., Craine, J. M., McLauchlan, K. \& Schimel, J. P. Litter quality and the 583 temperature sensitivity of decomposition. Ecology 86, 320-326 (2005).

58462 Conant, R. T. et al. Temperature and soil organic matter decomposition rates - synthesis 585 of current knowledge and a way forward. Glob. Change Biol. 17, 3392-3404 (2011).

58663 Smith, V. C. \& Bradford, M. A. Do non-additive effects on decomposition in litter-mix 587 experiments result from differences in resource quality between litters? Oikos 102, 235$588 \quad 242(2003)$.

58964 Gelman, A. Scaling regression inputs by dividing by two standard deviations. Stat. in $590 \quad$ Med. 27, 2865-2873 (2008).

59165 Baayen, R. H., Davidson, D. J. \& Bates, D. M. Mixed-effects modeling with crossed 592 random effects for subjects and items. J. Mem. Lang. 59, 390-412 (2008).

59366 Nakagawa, S. \& Schielzeth, H. A general and simple method for obtaining R2 from generalized linear mixed-effects models. Meth. Ecol. Evol. 4, 133-142 (2013).

596 Acknowledgements

597 Thanks to Rebecca Pas and Maria Hundscheid for lab assistance. Research was supported by

598 grants to MAB from the U.S. National Science Foundation (DEB-1457614), The Royal

599 Netherlands Academy of Arts and Sciences (Visiting Professors Programme), and the

600 Netherlands Production Ecology \& Resource Conservation Programme for Visiting Scientists. 
601 GFV was supported by an NWO-VENI from the Netherlands Organisation for Scientific

602 Research (863.14.013). MM-F and WHP were supported by a European Research Council grant

603 (ERC-Adv 260-55290), and GTF by grant EC2CO-Multivers. Thanks to the Bradford lab group

604 for comments on an earlier version of the script.

605

606 Authorship

607 MAB and GFV contributed equally to this work. They designed the study, co-wrote the

608 manuscript, constructed litterbags and carried out the lab analyses. All authors established,

609 maintained and collected data from the field sites. MAB, GFV, DSM and SAW analysed data.

610 All authors contributed to data interpretation and paper writing.

611

612 Additional information

613 Supplementary information is available for this paper.

614 Correspondence and requests for materials should be addressed to M.A.B.

615

616 Competing interests

617 The authors declare no competing financial interests.

618

619 Figure 1 Study design and site characteristics. Spatial organisation and operational

620 definitions of the study extent and observational grain are given in the hierarchical figure (site to

621 microsite). Sites are named for the closest city and their climate data are from climatedata.eu for

622 the months (May-June) of litterbag incubation, giving the range across months in the average

623 high and low temperature and precipitation. Soil data are the mean soil temperature and litter 
624 moisture data measured across the study period. Latitude and longitude data are for one transect

625 in each site.

626 Figure 2 Competing assumptions for how decomposer communities affect relationships

627 between climate and decomposition rates at regional to global scales. Ecosystem theory

628 holds that soil decomposer communities influence functional relationships between controls and

629 decomposition rates in a spatially invariant manner. For example, broad-scale patterns among

630 site-mean climate conditions are representative of a common relationship operating at finer

631 spatial scales (a): the assumption of scale invariance. Increasingly there is empirical evidence

632 that decomposer communities can be functionally distinct, meaning that broad-scale patterns

633 may instead emerge from distinct fine-scale (in this case within-site) relationships (b): the

634 assumption of scale dependence.

635 Figure 3 Measured variation in decomposition rates and controlling variables within and

636 among sites. The response variable (decomposition) is shown in (a), litter quality in (b), climate

637 variables in (c) and (d), soil nitrogen availability in (e) and an estimate of the active decomposer

638 biomass in (f). Points represent individual observations $(n=303)$ and are jiggered around the site

639 number to help prevent similar observations obscuring one another. Sites are described in Fig. 1.

$640 \quad$ Figure 4 | Estimated effects of temperature and moisture controls on decomposition rates.

641 Effect sizes are estimated for temperature (a) and moisture (b) using the coefficients from the

642 models presented in Table 1. Specifically, these coefficients were used in a regression equation,

643 along with the mean value across all 168 quadrats for the controls not under test, and then for the

644 control under test by systematically increasing the control from the lowest to highest observed

645 values across the 168 quadrats. Comparisons of effect sizes between the Microclimate versus

646 Site-mean climate models test whether patterns between site-mean climate and decomposition 
647 rates (effect sizes from the Site-mean climate model) approximate those operating at the

648 microsite scales at which decomposer organisms perceive the environment (effect sizes from the

649 Microclimate model). The temperature-decomposition relationship appears scale invariant

650 whereas the moisture-decomposition relationship is scale dependent (Fig. 2). The two Microsite

651 models ask whether inclusion of microbial biomass and $\mathrm{N}$ availability as additional variables

652 alters the estimated effects of temperature and moisture. Their inclusion does not appear to

653 strongly affect the climate-decomposition relationships.

654 Figure 5 Estimated effects of controls on decomposition rates. Effect sizes are estimated

655 from the Microsite interactions model presented in Table 1, and in (b) also from the Microsite

656 main effects model, following the procedure described in the legend of Fig. 4. In (a), plots for

657 each variable are generated using unstandardized coefficients from the "Microsite interactions"

658 model and the measured range in microsite conditions. The levels of each variable are

659 relativized, ranging from the minimum (0\%) to maximum (100\%) measured value, revealing that

660 microbial biomass (Microbe) has an effect size approximately equivalent to both temperature and

661 moisture. In (b), comparison of the two models asks whether the effect size of the microbial

662 biomass is additive or non-additively dependent on the other controlling variables. Its effect

663 seems primarily additive, given the similarity in the two plots. However, the effect sizes plotted

664 in (c) and (d) reveal that this additive effect of microbial biomass can still strongly determine

665 temperature and moisture effects on decomposition rates. The level of microbial biomass is

666 relativized, with five values shown ranging from the minimum (0\%) to maximum (100\%)

667 observed value. There are much stronger absolute decomposition responses to temperature and

668 moisture when microbial biomass values are greater.

669 
670 Table 1 Coefficients, significance and $r^{2}$ values for the linear mixed models used to evaluate 671 controls on litter decomposition rates. 


\section{Operational definitions of scale and variance}

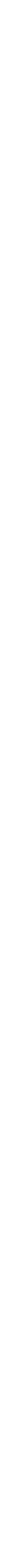




\section{(a) Assumption of scale invariance}

The among-site mean climateprocess relationship (solid line) approximates the slope of the within-site microclimate-process relationships (dotted lines)

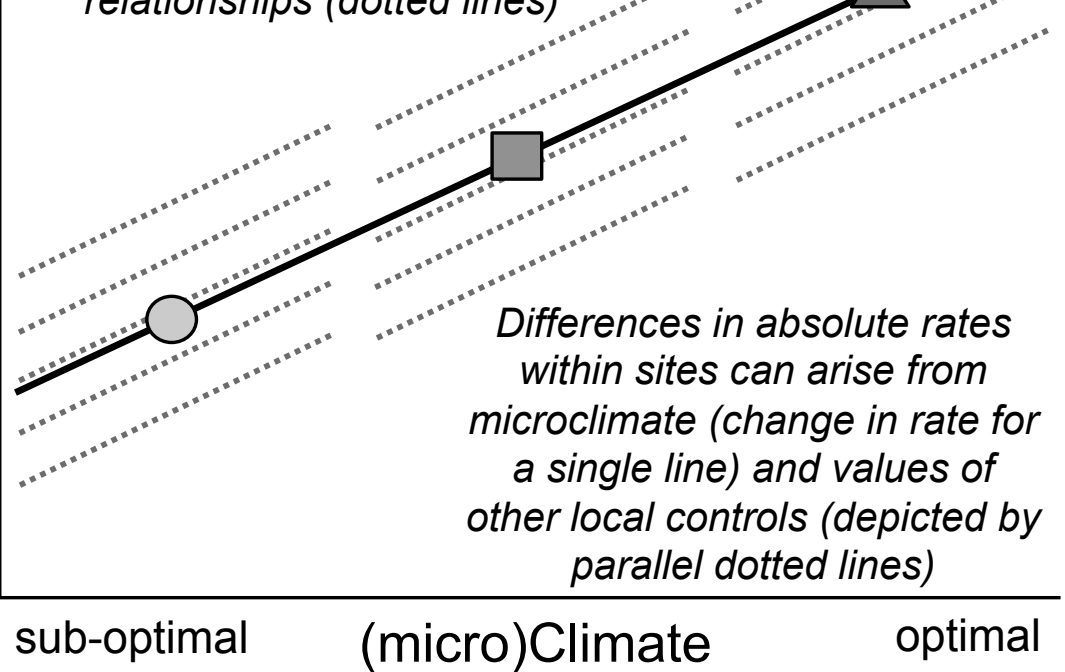

high

(b) Competing assumption of scale dependence

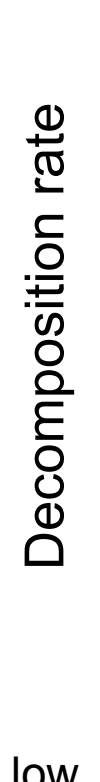

low

sub-optimal (micro)Climate optimal




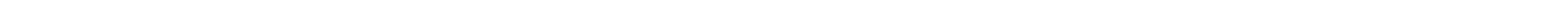


Table 1 Coefficients, significance and $r^{2}$ values for the linear mixed models used to evaluate controls on litter decomposition rates ${ }^{1}$. Shown in the second column are standardized coefficients for the full model, where "Microsite" refers to the level at which the variables were observed, and "interactions" to the inclusion of all 2-way interactions among the predictors.

Unstandardized coefficients were used when plotting Figs. 4, 5 and Supplementary Fig. 1. The consequence of aggregating microsite variation to generate "Site means" for the predictor variables was examined, but microsite variation in the response variable was retained to maintain the number of observations $(n=303)$. Significant $(P<0.05)$ and marginally-significant $(P<0.1)$ coefficients are shown in bold and italic fonts, respectively.

\begin{tabular}{|c|c|c|c|c|c|}
\hline \multirow[b]{3}{*}{ Variables } & \multicolumn{5}{|c|}{ Model } \\
\hline & \multirow[b]{2}{*}{$\begin{array}{c}\text { Microsite } \\
\text { interactions }\end{array}$} & \multicolumn{4}{|c|}{ Unstandardized coefficients } \\
\hline & & $\begin{array}{c}\text { Microsite } \\
\text { interactions }\end{array}$ & $\begin{array}{c}\text { Microsite main } \\
\text { effects }\end{array}$ & Microclimate & $\begin{array}{l}\text { Site-mean } \\
\text { climate }\end{array}$ \\
\hline Intercept & $27.0 \pm 0.689$ & $-70.0 \pm 14.629$ & $-17.1 \pm 6.264$ & $-15.6 \pm 6.365$ & $-24.1 \pm 6.960$ \\
\hline Litter N & $16.1 \pm 0.856$ & $45.3 \pm 5.998$ & $19.3 \pm 1.173$ & $19.2 \pm 1.198$ & $22.6 \pm 1.283$ \\
\hline Temperature & $-4.49 \pm 1.600$ & $5.03 \pm 1.344$ & $1.05 \pm 0.702$ & $1.73 \pm 0.681$ & $2.81 \pm 0.759$ \\
\hline Temp $^{2}$ & $-6.84 \pm 3.285$ & $-0.069 \pm 0.033$ & $-0.047 \pm 0.018$ & $-0.063 \pm 0.018$ & $-0.100 \pm 0.021$ \\
\hline Moisture & $7.23 \pm 1.256$ & $0.240 \pm 0.156$ & $0.141 \pm 0.023$ & $0.120 \pm 0.022$ & $0.017 \pm 0.028$ \\
\hline Soil N & $0.732 \pm 1.075$ & $0.151 \pm 0.158$ & $0.014 \pm 0.028$ & na & na \\
\hline Microbe & $4.59 \pm 1.165$ & $4.70 \pm 7.575$ & $4.93 \pm 1.477$ & na & na \\
\hline Lit $\times$ Temp & $-13.9 \pm 1.888$ & $-1.72 \pm 0.233$ & na & na & na \\
\hline Lit $\times$ Moist & $-0.275 \pm 2.057$ & $-0.007 \pm 0.049$ & na & na & na \\
\hline Lit $\times$ soilN & $1.58 \pm 1.666$ & $0.053 \pm 0.056$ & na & na & na \\
\hline Lit $\times$ Mic & $0.347 \pm 1.997$ & $0.535 \pm 3.077$ & na & na & na \\
\hline Temp $\times$ Moist & $-7.03 \pm 4.157$ & $-0.014 \pm 0.008$ & na & na & na \\
\hline Temp $\times$ soilN & $-3.09 \pm 2.035$ & $-0.009 \pm 0.006$ & na & na & na \\
\hline Temp $\times$ Mic & $-1.46 \pm 2.172$ & $-0.185 \pm 0.276$ & na & na & na \\
\hline Moist $\times$ soilN & $-3.02 \pm 2.536$ & $-0.002 \pm 0.001$ & na & na & na \\
\hline Moist $\times$ Mic & $4.55 \pm 2.923$ & $0.111 \pm 0.071$ & na & na & na \\
\hline soil $\mathrm{N} \times \mathrm{Mic}$ & $-0.409 \pm 1.226$ & $-0.014 \pm 0.042$ & na & na & na \\
\hline model $r^{2}$ & 66.3 & 66.3 & 57.1 & 55.2 & 57.6 \\
\hline
\end{tabular}

${ }^{1}$ Mean coefficients, their SD and significance are estimated using an MCMC sampling approach, and model $r^{2}$ values using a method that retains the random effects structure (see Methods). Model $r^{2}$ values were identical for the fixed and full (i.e. fixed + random) effects. 
Note: In the standardized Microsite interactions model, all sqrt VIFs were $<2$ except Temperature $^{2}$ which was 2.98 and Temperature $\times$ Moisture which was 2.30 . In the unstandardized Microsite interactions model, all sqrt VIFs were $<10$ except Temperature which was 16.0 and Temperature ${ }^{2}$ which was 14.8 .

In the unstandardized Microsite main effects model, all sqrt VIFs were $<2$ except Temperature and Temperature ${ }^{2}$; and the same was observed with the Microclimate model, and the Site-mean climate model.

na $=$ not applicable 